The Astrophysical Journal, 530:L57-L60, 2000 February 20

(C) 2000. The American Astronomical Society. All rights reserved. Printed in U.S.A.

\title{
PRIMORDIAL LITHIUM AND BIG BANG NUCLEOSYNTHESIS
}

\author{
Sean G. Ryan, ${ }^{1}$ Timothy C. Beers, ${ }^{2}$ Keith A. Olive, ${ }^{3}$ Brian D. Fields, ${ }^{4}$ and John E. Norris \\ Received 1999 September 23; accepted 1999 December 20; published 2000 January 21
}

\begin{abstract}
Recent determinations of the abundance of the light-element $\mathrm{Li}$ in very metal-poor stars show that its intrinsic dispersion is essentially zero and that the random error in the estimated mean $\mathrm{Li}$ abundance is negligible. However, a decreasing trend in the $\mathrm{Li}$ abundance toward lower metallicity indicates that the primordial abundance of $\mathrm{Li}$ can be inferred only after allowing for nucleosynthesis processes that must have been in operation in the early history of the Galaxy. We show that the observed Li versus $\mathrm{Fe}$ trend provides a strong discriminant between alternative models for Galactic chemical evolution of the light elements at early epochs. We critically assess current systematic uncertainties and determine the primordial Li abundance within new, much tighter limits: $(\mathrm{Li} / \mathrm{H})_{p}=1.23_{-0.32}^{+0.68} \times 10^{-10}$. We show that the Li constraint on $\Omega_{B}$ is now limited as much by uncertainties in the nuclear cross sections used in big bang nucleosynthesis (BBN) calculations as by the observed abundance itself. A clearer understanding of systematics allows us to sharpen the comparison with ${ }^{4} \mathrm{He}$ and deuterium and the resulting test of BBN.
\end{abstract}

Subject headings: cosmology: theory — Galaxy: halo — nuclear reactions, nucleosynthesis, abundances — stars: Population II

In the standard hot big bang nucleosynthesis (BBN) model (see, e.g., Olive, Steigman, \& Walker 2000), the primordial abundances of ${ }^{1} \mathrm{H},{ }^{2} \mathrm{H},{ }^{3} \mathrm{He},{ }^{4} \mathrm{He}$, and ${ }^{7} \mathrm{Li}$ fix the baryon content of the universe via the baryon-to-photon ratio, $\eta$. A crucial test of $\mathrm{BBN}$ is the concordance between the observationally inferred primordial abundances of the light elements. However, all current estimates of these abundances involve significant uncertainties. Several years ago, it appeared that estimates of the ${ }^{4} \mathrm{He}$ primordial mass fraction $Y_{p}$ had settled around $Y_{p}=$ $0.230 \pm 0.005$ (Pagel et al. 1992), but recently several authors have argued that there exist systematics that affect its derived abundance (e.g., stellar He I absorption) and result in a higher value, near 0.245 (Izotov \& Thuan 1998). For deuterium, quasar absorption line measurements give both "low" abundances around $\mathrm{D} / \mathrm{H}=(3-5) \times 10^{-5}$ (Burles \& Tytler 1998a, 1998b) and "high" values around $\mathrm{D} / \mathrm{H}=(15-25) \times 10^{-5}$ (Webb et al. 1997; Tytler et al. 1999). Recently, new high signal-to-noise ratio observations of $\mathrm{Li}$ in a carefully selected subset of very low metallicity stars have substantially reduced the observation errors in its determination (Ryan, Norris, \& Beers 1999, hereafter RNB), which has prompted us to examine carefully the random and systematic uncertainties associated with the estimation of the primordial abundance of ${ }^{7} \mathrm{Li}$ and its interpretation with respect to BBN.

In the inference of the primordial ${ }^{7} \mathrm{Li}$ abundance from observations of lithium in metal-poor stars, systematic errors arise from several sources, in particular (1) Galactic chemical evolution (GCE) of $\mathrm{Li}$, prior to a given star's birth from the interstellar medium, (2) corrections for possible depletion of a star's initial surface Li abundance, (3) derivation of the abundance itself, and (4) the presence of anomalous stars in a given

\footnotetext{
${ }^{1}$ Department of Physics, Open University, Walton Hall, Milton Keynes, MK7 6AA, England, UK.

${ }^{2}$ Department of Physics and Astronomy, Michigan State University, East Lansing, MI 48824-1116.

${ }^{3}$ Theoretical Physics Institute, School of Physics and Astronomy, University of Minnesota, 116 Church Street, SE, Minneapolis, MN 55455.

${ }^{4}$ Department of Astronomy, University of Illinois at Champaign-Urbana, 1002 West Green Street, Urbana, IL 61801.

${ }^{5}$ Research School of Astronomy and Astrophysics, Australian National University, Private Bag, Weston Creek Post Office, Canberra, ACT 2611, Australia.
}

sample. We examine each factor and summarize its impact on estimates of primordial ${ }^{7} \mathrm{Li}$. Progress toward a realistic estimate of the primordial abundance of ${ }^{7} \mathrm{Li}$ requires extremely precise measurements of $\mathrm{Li}$ abundance, so we focus on the recent observations of RNB, which are several times more precise than previous surveys and are derived from a sample of stars with homogeneously determined $\mathrm{Li}$ and $\mathrm{Fe}$ abundances.

GCE of ${ }^{7} \mathrm{Li}$ was long assumed to be negligible for metalpoor stars in view of its apparent uniformity (Spite \& Spite 1982). However, the existence of at least some GCE contribution to ${ }^{7} \mathrm{Li}$ from Galactic cosmic-ray (GCR) reactions had been inferred from observations of Be. Furthermore, the data of RNB exhibit a small but statistically significant increase of $\mathrm{Li}$ with $[\mathrm{Fe} / \mathrm{H}], 0.118 \pm 0.023(1 \sigma)$ dex per dex. The existence of a correlation between $\mathrm{Li}$ and $[\mathrm{Fe} / \mathrm{H}]$ has been reported several times previously (Norris, Ryan, \& Stringfellow 1994; Thorburn 1994; Ryan et al. 1996), at a similar level as reported by RNB but at considerably lower statistical significance because of the limitations in the observational determination of $\mathrm{Li}$ abundance. Bonifacio \& Molaro (1997) have even argued that this correlation is not present at all, but RNB show that this claim is likely to have been influenced by their adoption of incorrect $[\mathrm{Fe} / \mathrm{H}]$ values for a number of stars in their sample. New observations of $\mathrm{Li}$ in additional stars of very low metallicity by Spite et al. (2000), with $[\mathrm{Fe} / \mathrm{H}]$ taken from the revised calibration of Beers et al. (1999), confirm the claimed correlation of RNB.

The observed $\mathrm{Li}-\mathrm{Fe}$ trend shows that GCE cannot be ignored in the estimation of primordial ${ }^{7} \mathrm{Li}$, implying that $\mathrm{Li} \mathrm{GCE}$ can be constrained empirically. RNB explored Li production via a regression in logarithmic abundances:

$$
A(\mathrm{Li})=\alpha+\beta[\mathrm{Fe} / \mathrm{H}]
$$

where the lithium abundance $A(\mathrm{Li}) \equiv \log (\mathrm{Li} / \mathrm{H})+12.00$, and $[\mathrm{Fe} / \mathrm{H}] \equiv \log (\mathrm{Fe} / \mathrm{H})_{\text {star }}-\log (\mathrm{Fe} / \mathrm{H})_{\odot}$ measures the metallicity. They obtained $\beta=0.07-0.16$, depending on the adopted $[\mathrm{Fe} / \mathrm{H}]$ values, the errors in the $\mathrm{Li}$ abundance determination, and on whether some outlying points are excluded from the fit. 
TABLE 1

Inferred Primordial Lithium Abundance: Observed (RNB) Abundance is $\langle A(\mathrm{Li})\rangle_{-2.8}=2.12 \pm 0.02$

\begin{tabular}{|c|c|c|}
\hline Corrections to Apply Logarithmically & Value & Estimated Uncertainty \\
\hline \multicolumn{3}{|l|}{ (1) GCE/GCR: } \\
\hline Previous analyses (RNB) & -0.14 to -0.05 & \\
\hline Log data fit (eq. [1]) .... & -0.20 to -0.09 & \\
\hline Linear data fit (eq. [2]) & -0.12 to -0.04 & \\
\hline Linear data fit (eq. [3]) & -0.16 to -0.05 & \\
\hline Model fits (eqs. [2]-[3]) & -0.05 to -0.04 & \\
\hline Adopted (excludes model) & & $-0.11_{-0.09}^{+0.07}$ \\
\hline (2) Stellar depletion & & $+0.02_{-0.02}^{+0.08}$ \\
\hline (3a) $T_{\text {eff }}$-scale zero point ... & & $+0.08 \pm 0.08$ \\
\hline (3b) One-dimensional atmosphere models ...... & & $+0.00_{-0.00}^{+0.10}$ \\
\hline (3c) Convective treatment ............... & & $+0.00_{-0.00}^{+0.08}$ \\
\hline (3d) Non-LTE $\ldots \ldots \ldots \ldots \ldots$ & & $-0.02 \pm 0.01$ \\
\hline (3e) $g f$-values & & $+0.00 \pm 0.04$ \\
\hline (4) Anomalous objects & & $+0.00 \pm 0.01$ \\
\hline Total $\ldots \ldots \ldots \ldots \ldots \ldots \ldots \ldots \ldots \ldots \ldots \ldots \ldots \ldots \ldots \ldots \ldots \ldots \ldots$ & & $-0.03_{-0.13}^{+0.19}$ \\
\hline Inferred $A\left(\mathrm{Li}_{p}\right) \ldots \ldots \ldots \ldots \ldots \ldots \ldots \ldots \ldots$ & & $+2.09_{-0.13}^{+0.19}$ \\
\hline
\end{tabular}

NotE. - The weighted mean and 95\% CL uncertainty of observed Li abundances for a very metal-poor sample of halo main-sequence turnoff stars $(\mathrm{RNB})$ with $\langle[\mathrm{Fe} / \mathrm{H}]\rangle=-2.8$ and the corrections required to deduce the primordial value.

In the present work, we investigate a fitting form that better follows Li production by GCE and that simplifies extrapolation to the primordial value. $\mathrm{Li}$ production is proportional to the cumulative number, $N_{\mathrm{SN}}$, of Type II supernovae, as these are both GCR accelerators and the site of the $\nu$-process (Woosley et al. 1990) that also produces ${ }^{7} \mathrm{Li}$. At present, it is unclear whether iron or oxygen provides the better tracer of $N_{\mathrm{SN}}$; thus, we will perform fits with both. If the cumulative number of supernovae is best reflected by iron $\left(N_{\mathrm{SN}} \propto \mathrm{Fe}\right)$, then a fit to linear abundance scales is appropriate:

$$
\mathrm{Li} / \mathrm{H}=a^{\prime}+b^{\prime} \mathrm{Fe} / \mathrm{Fe}_{\odot} \text {. }
$$

Here $a^{\prime}$ directly measures the primordial ${ }^{7} \mathrm{Li}$ abundance, while $b^{\prime}$ probes GCE. The linear fit parameters are sensitive to systematic Li errors; a change by $\Delta_{\text {cal }}$ dex in the log shifts both

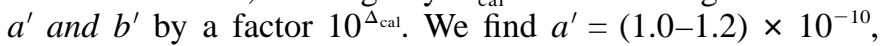
corresponding to $A\left(\mathrm{Li}_{p}\right)=2.00-2.08$ and slopes $b^{\prime}=(40-$ $180) \times 10^{-10}$. If, on the other hand, oxygen (which is more

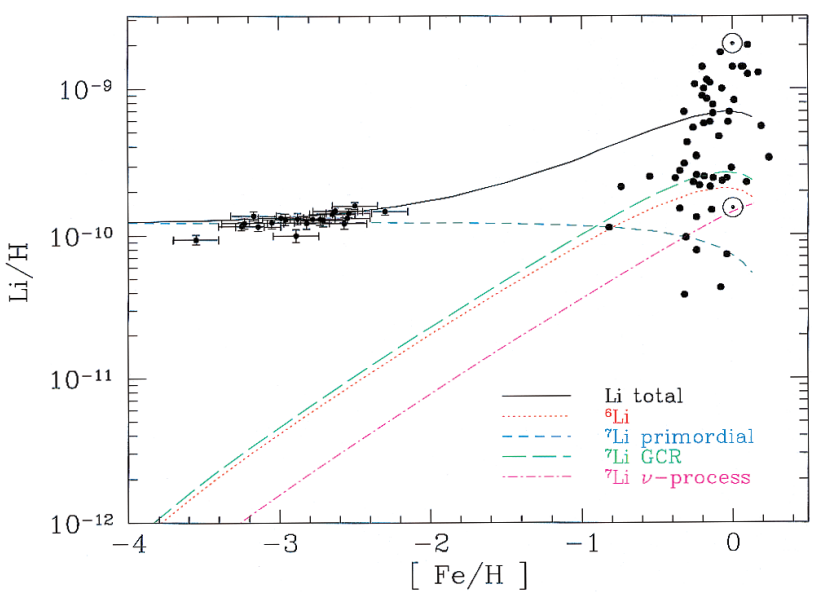

FIG. 1.-Contributions to the total predicted lithium abundance from the adopted GCE model of Fields \& Olive (1999a, 1999b), compared with lowmetallicity stars (RNB) and high-metallicity stars (Lambert, Heath, \& Edvardsson 1991). The solid curve is the sum of all components. difficult to measure than iron) is a better tracer of Type II supernovae than iron, then $N_{\mathrm{SN}} \propto \mathrm{O}$, and we expect

$$
\mathrm{Li} / \mathrm{H}=a+b \mathrm{O} / \mathrm{O}_{\odot}
$$

Recent observations show $\mathrm{O} / \mathrm{O}_{\odot}=\left(\mathrm{Fe} / \mathrm{Fe}_{\odot}\right)^{1+\omega}$, with $\omega=$ - 0.31 (Israelian, García-López, \& Rebolo 1998; Boesgaard et al. 1999). In this case, the data indicate $a=(0.9-1.2) \times$ $10^{-10}$ and $b=(9-34) \times 10^{-10}$.

Four empirical estimates of the logarithmic correction for the GCE contribution to the mean $\langle A(\mathrm{Li})\rangle$ in the RNB sample appear in Table 1. These are based on the previous analysis of RNB (using both logarithmic fits and the observed ${ }^{6} \mathrm{Li} /{ }^{7} \mathrm{Li}$ ratio), logarithmic fits (eq. [1]), and the linear fits (eq. [2]). The adopted correction is $-0.11_{-0.09}^{+0.07}$ in the log and spans the range of these four. The correction derived from a theoretical model, discussed below, is indicated only for comparison. The adopted correction for GCE is derived in an entirely empirical way.

The observed Li trend versus metallicity can also be used to constrain Galactic production mechanisms. Post-BBN sources of lithium in the oldest (Population II) stars are the GCR nucleosynthesis of ${ }^{6} \mathrm{Li}$ and ${ }^{7} \mathrm{Li}$ and the supernova $\nu$-process (Woosley et al. 1990) that produces ${ }^{7} \mathrm{Li}$ and ${ }^{11} \mathrm{~B}$. To illustrate the expected $\mathrm{Li}-\mathrm{Fe}$ trend, we have calculated $\mathrm{Li}$ evolution within a simple, one-zone (closed-box) GCE model (Fields \& Olive 1999a, 1999b). The model accounts for the decrease in the primordial component of ${ }^{7} \mathrm{Li}$ due to astration at high metallicity and for the increase of $\mathrm{Li}$ at low metallicities due to Galactic sources of Li, namely, GCR and stellar nucleosynthesis. The GCR model assumes that cosmic rays are accelerated by supernovae; the GCR nucleosynthesis parameters are set by present cosmic-ray properties and the meteoritic $\mathrm{Be}$ abundance. The stellar nucleosynthesis contribution to $\mathrm{Li}$ is the $\nu$-process, which is fixed by its contribution to the ${ }^{11} \mathrm{~B}$ meteoritic abundance. To reproduce the observed $\mathrm{O}-\mathrm{Fe}$ scaling accurately, we derive $\mathrm{Fe}$ from the calculated $\mathrm{O}$ evolution via $[\mathrm{O} / \mathrm{Fe}]=\omega[\mathrm{Fe} / \mathrm{H}]($ with $\omega=-0.31)$. With these normalizations, the modeled evolution of $\mathrm{Be}$ and $\mathrm{B}$ fits the available Population II observations, and the GCE of ${ }^{6} \mathrm{Li}$ and ${ }^{7} \mathrm{Li}$ are fixed.

Figure 1 shows the different Li components for the model 
with ${ }^{7} \mathrm{Li}_{p}=1.23 \times 10^{-10}$. Note that the fit is good at low metallicity but poor at $[\mathrm{Fe} / \mathrm{H}]$ near solar, where additional stellar production (and destruction) mechanisms of ${ }^{7} \mathrm{Li}$ are required (e.g., Matteucci, D’Antona, \& Timmes 1995) but are expected to be unimportant for the lowest metallicity objects. We fit the model by regressions of equations (1)-(3) over the metallicity range of the RNB data $(-3.5 \leq[\mathrm{Fe} / \mathrm{H}] \leq-2.3)$, and we find that $b=12.6 \times 10^{-10}, b^{\prime}=65 \times 10^{-10}$, and $\beta=0.037-0.074$ [for input ${ }^{7} \mathrm{Li}_{p}=(0.9-1.9) \times 10^{-10}$ ]. The model's "linear slopes," $b$ and $b^{\prime}$, are independent of the input ${ }^{7} \mathrm{Li}_{p}$, while its "log slope," $\beta$, does depend on ${ }^{7} \mathrm{Li}_{p}$. The GCE model gives slopes that fall within the observed ranges. This demonstrates that the observed GCE effects are consistent with the expected trends. Using these model slopes, we compute the inferred deviation of the primordial value to the weighted mean at $[\mathrm{Fe} / \mathrm{H}]=-2.8$ to be -0.05 to $-0.04 \mathrm{dex}$.

Note that the models only include Galactic Li production due to GCRs and the $\nu$-process. The observed Li evolution can also constrain possible additional Li sources, such as those suggested to produce primary $\mathrm{Be}$ along with $\mathrm{Li}$, or stellar Li production (e.g., Higdon, Lingenfelter, \& Ramaty 1998; Vangioni-Flam, Cassé, \& Audouze 2000 and references therein) and would increase the predicted slope.

Stellar interiors burn Li and alter its surface abundance, unless $\mathrm{Li}$ is preserved in the thin outer layer of the atmosphere containing a few percent of the star's mass. In situ depletion of $\mathrm{Li}$ has long been regarded as the major systematic uncertainty for inference of $A\left(\mathrm{Li}_{p}\right)$ from present-day observed abundances. Stellar evolution models predict the depletion factors. The simplest models imply almost no destruction $(<0.05$ dex, possibly $\lesssim 0.01 \mathrm{dex}$ ) in very metal-poor turnoff stars (Deliyannis, Demarque, \& Kawaler 1990). Models incorporating rotationally induced mixing had predicted depletion factors of $\sim 1 \mathrm{dex}$, although more recent efforts give lower values of $\sim 0.2-0.4$ dex (Pinsonneault et al. 1999) and, importantly, predict the existence of star-to-star differences in observed Li abundances because of the range of stellar rotation and other intrinsic stellar properties to which the models have some sensitivity. RNB's observation of a negligible intrinsic spread in Li for very metalpoor turnoff stars, $\sigma_{\text {int }}<0.02$ dex, rules out rotational depletion even as low as 0.1 dex. As diffusion is also absent (Ryan et al. 1996), we conclude that in situ depletion is of minor importance $(<0.1 \mathrm{dex}$ and possibly as little as $\sim 0.01 \mathrm{dex})$.

A stellar Li abundance is a derived quantity, obtained via a physical parameter-dependent and model-dependent analysis of a stellar spectrum, the uncertainties of which also affect estimates of $A\left(\mathrm{Li}_{p}\right)$. Effective temperature calibrations for stellar atmospheres can differ by up to $150-200 \mathrm{~K}$, with higher temperatures resulting in estimated $\mathrm{Li}$ abundances that are higher by 0.065 dex per $100 \mathrm{~K}$. The scale initially adopted by RNB gives temperatures that are cooler than a recent calibration (Alonso, Arribas, \& Martinez-Roger 1996) by $\simeq 120 \mathrm{~K}$. We now adjust the abundances (Table 1) to the latter calibration, but note that systematic errors of $\pm 120 \mathrm{~K}$ may still exist. This is one of the largest contributions to the uncertainty in $A\left(\mathrm{Li}_{p}\right)$. Fortunately, errors in the derived $\mathrm{Li}$ abundance that depend on the adopted surface gravity, microturbulence, and damping parameters of the $\mathrm{Li}$ line are negligible.

Concerns about the adoption of one-dimensional, planeparallel model atmospheres in the analysis have been reduced by simulations of solar-type granulation (Uitenbroek 1998), which show that the $\mathrm{Li}$ abundance is underestimated in the onedimensional approximation by less than 0.10 dex (and possibly less than $0.01 \mathrm{dex}$ ), depending on the theoretical prescription for microturbulence. Consistent results in the metal-poor star HD 140283 (Bonifacio \& Molaro 1998) from the lines Li $\lambda 6104$ and $\lambda 6707$ inspire further confidence. However, the new threedimensional hydrodynamical simulations of Asplund et al. (1999) suggest that one-dimensional models may overestimate the $\mathrm{Li}$ abundances of metal-poor stars by $0.2-0.35 \mathrm{dex}$. We refrain from applying this correction because of the preliminary nature of that work but note that a future reassessment may be required. Among one-dimensional models, those with greater convective flux can lead to $\mathrm{Li}$ abundances higher by $0.08 \mathrm{dex}$ (Ryan et al. 1996). Corrections for non-LTE are only -0.01 to -0.03 dex (Carlsson et al. 1994), and uncertainty in the $g f$ values' use for the estimation of $\mathrm{Li}$ abundance contributes an additional uncertainty of only $0.02 \operatorname{dex}(1 \sigma)$ (Thorburn 1994).

Apart from the grossly Li-depleted star G186-26, only one other star in the RNB sample was rejected by outlier-detection algorithms, changing the mean abundance by a mere $\sim 0.005$ dex. We adopt 0.01 dex as the uncertainty arising from such objects. Other very Li-poor (and one Li-rich) halo field dwarfs that are known constitute only a few percent of the field population and, most importantly, appear unrepresentative of the vast majority of field halo turnoff stars. The reasons for their anomalous abundances remain of great interest, but their existence need not prevent us discussing the vast majority of "normal" stars separately in this work.

The situation may be different in halo globular clusters, as Boesgaard et al. (1998) find a range of 0.5 dex in technically challenging observations of seven stars in M92. The contrast between the small Li spread of the field halo dwarfs and the larger spread in M92 may indicate that they have undergone different processes, related to the stellar densities at which they formed. Moreover, globular cluster stars sample only a tiny region of the Galactic halo compared with the field stars. The latter should therefore provide a more reliable measure of the chemical evolution of the Galaxy, as opposed to the chemical evolution of a small region of space with high stellar density.

Estimated primordial abundances of the light elements fix the one free parameter of the standard BBN model, $\eta$. Table 1 summarizes all of the corrections needed to infer the primordial Li abundance from the weighted mean of the RNB observations. The various error estimates, which include random and systematic uncertainties, are clearly non-Gaussian, so combining them is an imprecise and subjective process. We take quadratic sums for the positive and negative uncertainties separately, and we regard these as estimates of the $95 \%$ confidence limits (CLs). We infer a primordial abundance $A\left(\mathrm{Li}_{p}\right)=2.09_{-0.13}^{+0.19}\left[(\mathrm{Li} / \mathrm{H})_{p}=1.23_{-0.32}^{+0.68} \times 10^{-10}\right]$, where the errors incorporate statistical (negligible) and systematic (more significant) effects. ${ }^{6}$ These errors are now sufficiently small that the theoretical inputs (i.e., the nuclear cross-sectional uncertainties, and the fact that ${ }^{7} \mathrm{Li}-\eta$ is near its minimum and thus slowly varying) contribute as much to the range of corresponding $\eta$ values as does the observed abundance of $\mathrm{Li}$.

BBN concordance is best examined by establishing likelihood distributions (as a function of $\eta$ ) for each element, convolving the theoretical and observational uncertainties (Fields et al. 1996). Figure 2 shows the likelihood distributions for ${ }^{4} \mathrm{He}$ and four possible values of the primordial ${ }^{7} \mathrm{Li}$ abundance, all of which give excellent agreement. For $\left({ }^{7} \mathrm{Li} / \mathrm{H}\right)_{p}=1.6 \times$ $10^{-10}\left[A\left(\mathrm{Li}_{p}\right)=2.20\right]$, there are two likely values of $\eta_{10} \equiv$ $10^{10} \eta=1.9$ and 3.6, because the predictions are not monotonic in $\left({ }^{7} \mathrm{Li} / \mathrm{H}\right)_{p}$. For $\left({ }^{7} \mathrm{Li} / \mathrm{H}\right)_{p} \lesssim 1.1 \times 10^{-10}\left[A\left(\mathrm{Li}_{p}\right) \lesssim 2.04\right]$, the

${ }^{6}$ The weighted mean differs slightly from the robust mean, viz., 2.11 . 


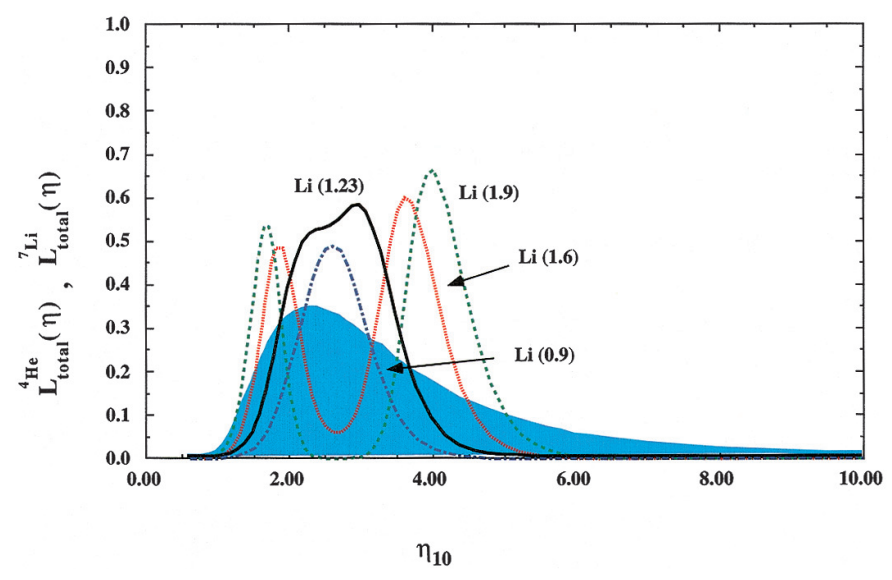

FIG. 2.-Likelihood distributions for four values of primordial $\left({ }^{7} \mathrm{Li} / \mathrm{H}\right)_{p}$ $\left[10^{10} \times{ }^{7} \mathrm{Li} / \mathrm{H}=1.9\right.$ (dashed curve), 1.6 (dotted curve), 1.23 (solid curve), and 0.9 (dash-dotted curve)] and for ${ }^{4} \mathrm{He}_{p}$ (shaded blue region) for which we adopt $Y_{p}=0.238 \pm 0.002 \pm 0.005$ (random and systematic uncertainties; Fields \& Olive 1998).

$\mathrm{Li}$ abundance is at or below the BBN-predicted value, so there is only one peak, at $\eta_{10} \simeq 2.6$; uncertainties in the prediction and observation prevent the likelihood function from vanishing. The peaks of the combined distribution [the product of $L_{{ }^{4} \mathrm{He}}(\eta)$ and $L_{7_{\mathrm{Li}}}(\eta)$; not shown] are at roughly the same value of $\eta$ as in the individual $L_{7_{\mathrm{Li}}}(\eta)$ distributions.

Overall concordance (at 95\% CL) occurs for $\eta_{10} \equiv$ $10^{10} \eta=(1.4-4.9), \quad(1.5-4.4),(1.7-3.9)$, and $(1.8-3.6)$, for $10^{10} \times\left({ }^{7} \mathrm{Li} / \mathrm{H}\right)_{p}=1.9,1.6,1.23$, and 0.9 , respectively. The baryon density corresponding to $\eta=(1.7-3.9) \times 10^{-10}$ is
$\Omega_{B}=(0.025-0.057) / h_{50}^{2}$ (where $h_{50}$ is the Hubble constant in units of $50 \mathrm{~km} \mathrm{~s}^{-1} \mathrm{Mpc}^{-1}$ ). We can then use this result to assess the diverse primordial deuterium values that have been reported in the recent literature. For high $(\mathrm{D} / \mathrm{H})_{p}\left(2.0 \times 10^{-4}\right)$, the peak of the $(\mathrm{D} / \mathrm{H})_{p}$ likelihood function is at $\eta_{10}=1.7(95 \% \mathrm{CL}=$ 1.4-3.8), in very good agreement with the results from ${ }^{4} \mathrm{He}_{p}$ and $\left({ }^{7} \mathrm{Li} / \mathrm{H}\right)_{p}$. For low $(\mathrm{D} / \mathrm{H})_{p}\left(3.4 \times 10^{-5}\right)$, the peak of the $(\mathrm{D} / \mathrm{H})_{p}$ likelihood function is at $\eta_{10}=5.2(95 \% \mathrm{CL}=4.6-6.1)$, which would require $\left({ }^{7} \mathrm{Li} / \mathrm{H}\right)_{p}$ at the upper end of the allowed range. However, if the low $(\mathrm{D} / \mathrm{H})_{p}$ value was even only slightly higher, at $5 \times 10^{-5}$ (Levshakov, Tytler, \& Burles 1998), then the $(\mathrm{D} / \mathrm{H})_{p}$ peak would occur at $\eta_{10}=4.0(95 \% \mathrm{CL}=3.6-$ 4.6), consistent with the ranges for ${ }^{4} \mathrm{He}_{p}$ and $\left({ }^{7} \mathrm{Li} / \mathrm{H}\right)_{p}$.

Additional precision observations of $\mathrm{Li}$ can sharpen both the estimation of the primordial abundance of this element and the derived constraints on GCE. New data for stars of extremely low metallicity, $[\mathrm{Fe} / \mathrm{H}]<-3$, can confirm or refute the $\mathrm{Li}-\mathrm{Fe}$ increase reported by RNB and will also minimize the extrapolation to the primordial abundance. Similarly precise Li data at higher metallicities, $[\mathrm{Fe} / \mathrm{H}]=-2$ to -1 , could help identify the onset of significant GCE Li production and the rise to the Population I level.

The authors gratefully acknowledge discussions with C. P. Deliyannis, M. Pinsonneault, and J. A. Thorburn on issues of stellar depletion. S. G. R. thanks the Institute of Astronomy of the University of Cambridge for provision of facilities following the closure of the Royal Greenwich Observatory. The work of K. A. O. was supported in part by DoE grant DE-FG0294ER-40823 at the University of Minnesota. T. C. B. acknowledges support from grant AST 95-29454 from the National Science Foundation.

\section{REFERENCES}

Alonso, A., Arribas, S., \& Martinez-Roger, C. 1996, A\&AS, 117, 227

Asplund, M., Nordlund, Å., Trampedach, R., \& Stein, R. F. 1999, A\&A, 346, L17

Beers, T. C., Rossi, S., Norris, J. E., Ryan, S. G., \& Shefler, T. 1999, AJ, 117, 981

Boesgaard, A. M., Deliyannis, C. P., Stephens, A., \& King, J. R. 1998, ApJ, 493, 206

Boesgaard, A. M., King, J. R., Deliyannis, C. P., \& Vogt, S. S. 1999, AJ, 117, 492

Bonifacio, P., \& Molaro, P. 1997, MNRAS, 285, 847 1998, ApJ, 500, L175

Burles, S., \& Tytler, D. 1998a, ApJ, 499, 699

- 1998b, ApJ, 507, 732

Carlsson, M., Rutten, R. J., Bruls, J. H. M. J., \& Shchukina, N. G. 1994, A\&A, 288, 860

Deliyannis, C. P., Demarque, P., \& Kawaler, S. D. 1990, ApJS, 73, 21

Fields, B. D., Kainulainen, K., Olive, K. A., \& Thomas, D. 1996, NewA, 1, 77

Fields, B. D., \& Olive, K. A. 1998, ApJ, 506, 177

1999a, ApJ, 516, 797

1999b, NewA, 4, 255

Higdon, J. C., Lingenfelter, R. E., \& Ramaty, R. 1998, ApJ, 509, L33

Israelian, G., García-López, R. J., \& Rebolo, R. 1998, ApJ, 507, 805

Izotov, Y. I., \& Thuan, T. X. 1998, ApJ, 500, 188

Lambert, D. L., Heath, J. E., \& Edvardsson, B. 1991, MNRAS, 253, 610

Levshakov, S. A., Tytler, D., \& Burles, S. 1998, preprint (astro-ph/9812114)
Matteucci, F., D’Antona, F., \& Timmes, F. X. 1995, A\&A, 303, 460

Norris, J. E., Ryan, S. G., \& Stringfellow, G. S. 1994, ApJ, 423, 386

Olive, K. A., Steigman, G., \& Walker, T. P. 2000, Phys. Rep., in press (astro$\mathrm{ph} / 9905320$ )

Pagel, B. E. J., Simonson, E. A., Terlevich, R. J., \& Edmunds, M. G. 1992, MNRAS, 255, 325

Pinsonneault, M. H., Walker, T. P., Steigman, G., \& Narayanan, V. K. 1999, ApJ, 527, 180

Ryan, S. G., Beers, T. C., Deliyannis, C. P., \& Thorburn, J. A. 1996, ApJ, 458,543

Ryan, S. G., Norris, J. E., \& Beers, T. C. 1999, ApJ, 523, 654 (RNB)

Spite, F., \& Spite, M. 1982, A\&A, 115, 357

Spite, M., Cayrel, R., Beers, T. C., Nordstrom, B., Barbuy, B., Andersen, J., \& Nissen, P. E. 2000, in Galaxy Evolution: Connecting the Distant Universe with the Local Fossil Record, ed. M. Spite \& F. Crifo (Dordrecht: Kluwer), in press

Thorburn, J. A. 1994, ApJ, 421, 318

Tytler, D., Burles, S., Lu, L., Fan, X-M., Wolfe, A., \& Savage, B. D. 1999, AJ, 117, 63

Uitenbroek, H. 1998, ApJ, 498, 427

Vangioni-Flam, E., Cassé, M., \& Audouze, J. 2000, Phys. Rep., in press (astro$\mathrm{ph} / 9907171)$

Webb, J. K., et al. 1997, Nature, 388, 250

Woosley, S. E., Hartmann, D. H., Hoffman, R. D., \& Haxton, W. C. 1990, ApJ, 356, 272 